\title{
Letter to the Editor: The Significance of Medicinal Herbs for Treating Candidiasis
}

\author{
Mehrnaz Molaei and Mohamad Reza Havasian* \\ Department of Dentistry, Sechenov First Moscow State Medical University, Russia
}

*Corresponding author: Mohamad Reza Havasian, Department of Dentistry, Sechenov First Moscow State Medical University, Moscow, Russia

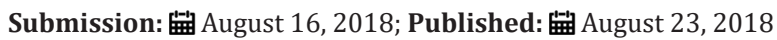

\section{Editorial}

Candida albicans grows in the growth medium or tissue as oval and budding yeast cells. Candidiasis is undoubtedly one of the most significant as well as most common opportunistic fungal diseases in humans. The infection is acute, sub-acute, or chronic that is often seen in the skin, nails, vaginal mucus, digestive system etc. The host's reaction against this disease ranges from brief itching and inflammation to the chronic form, acute suppurative or granulomatous. Candida albicans is the main cause of $85-90 \%$ of Vulvovaginal candidiasis cases [1]. In fact, vulvovaginitis is common in most of the women receiving medical cares and every year more than 10 million people suffer from this complication. It has been estimated that from every 10 women, at least seven of them suffer from a fungal infection at least once in their lifetime, and from every 10 women, 4 will suffer from repeated recurrence.

Nowadays, the widespread prevalence of opportunistic fungal disease in susceptible individuals and the growing increase of drug resistance and their adverse effects have resulted in the increasing importance of investigating the antifungal effects of plants [2]. Traditional treatments are both cheaper and more effective than modern treatments. In fact, in societies where medicinal herbs are widely used, there is a lower risk of infection with resistant pathogens. Numerous studies have been so far conducted on the effect of herbal extracts in traditional medicine for treating candidiasis. The medicinal herbs that have been so far investigated include hydro-alcoholic extract of Scrophularia striata, aqueous extract of Allium hirtifolium and alcoholic extract of Inner Stratum of Oak Fruit. Each of the abovementioned studies have resulted in various effects on different strains of candida albicans [3-5]. The general findings of these studies have indicated that the hydro-alcoholic extract of Scrophularia striata and Nectaroscordum Tripedale have little inhibitory effect on the isolates of candida albicans and the ineffectiveness of aqueous extract of Allium hirtifolium on these isolates. However, in the study conducted by Panahi et al. [6], it has been claimed that the alcoholic extract of Inner Stratum of Oak Fruit has strong inhibitory effect on the samples of candida albicans isolated from fungal vaginitis. As this plant is traditionally applied in the western areas of Iran and since its constituents are not clearly identified, it has been recommended to identify the constituents of this plant and evaluate the cytotoxic and MTT effects of this plant by conducting further studies.

Moreover, medicinal herbs are preferable over applying the common chemical drugs used for treating the infections; chemical drugs not only result in serious complications and medicinal resistance but also impose high costs on both families and societies. Furthermore, medicinal herbs are easier to be applied and more accepted by the society. In fact, traditional treatments are both cheaper and more effective than modern treatments. In societies where traditional treatments are applied, there is a lower risk of acquiring infections with resistant pathogens and there is a lower risk of acquiring hospital infections in comparison to societies where antibiotics are applied [7]. Furthermore, one of the methods for preventing antibiotic-resistant pathogens is applying medicinal herbs based on medicinal compounds. So far, extensive studies have been conducted to investigate the effects of different antifungal drugs on the strains of $\mathrm{C}$ sropicaeis, $\mathrm{C}$ parapsilosis, C gudliermondi and C kefyr. Given the desirable findings obtained from the previous studies, it is of increasing significance that researchers have more accurate studies about the structure of different herbal extracts and their cytotoxic and MTT effects.

\section{References}

1. Mohamadi J, Motaghi M, Panahi J, Havasian MR, Delpisheh A, et al. (2014) Anti-fungal resistance in candida isolated from oral and diaper rash candidiasis in neonates. Bioinformation 10(11): 667-670.

2. Judaki A, Panahi J, Havasian MR, Tajbakhsh P, Roozegar MA (2014) Study of the inhibitory effect of Quercus Coccifera's aqueous extract on Staphylococcus aureus and Pseudomonas aeruginosa in vitro. Bioinformation 10(11): 689-692.

3. Havasian MR, Panahi J, Pakzad I, Davoudian A, Jalilian A, et al. (2013) Study of Inhibitory effect of alcoholic and aqueous extract of Scrophularia striata (tashne dari) on candida albicans in vitro. Research in Medicine 36(5): 19-23. 
4. Panahi J, Havasiyan MR, Gheitasi S, Pakzad I, Jaliliyan A, et al. (2013) The in vitro Inhibitory Effects of the Aqueous Extracts of Summer Onion on Candida Albicans. J of Ilam Uni Med Sci 21(1): 54-59.

5. Panahi J, Havasian MR, Pakzad I, davudian A, Jalilian F, et al. (2013) In vitro inhibitory effect of alcoholic extract of inner stratum of oak fruit (Jaft) on Candida Albicans. J Pharm Biomed Sci 3(1): 5-8.

6. Panahi J, Tajbakhsh P, Judaki A, Havasian MR (2014) In vitro inhibitory effect of the hydro-alcoholic extract from the avicennia marina (Hara) on Candida Albicans. Mintage Journal of Pharmaceutical and Medical Sciences 3(Suppl 4): 9-11.

7. Roozegar MA, Pournazari Z, Nazari MR, Havasian MR, Panahi J, et al. (2014) In vitro inhibitory effect of alcoholic and aqueous extract of Umbilicus intermedius boiss (nafe venus) on Staphylococcus aureus and Pseudomonas aeruginosa. J of Evolution of Med and Dent Sci 3(31): 8686-8691.

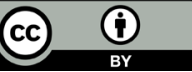

Creative Commons Attribution 4.0 International License

For possible submissions Click Here

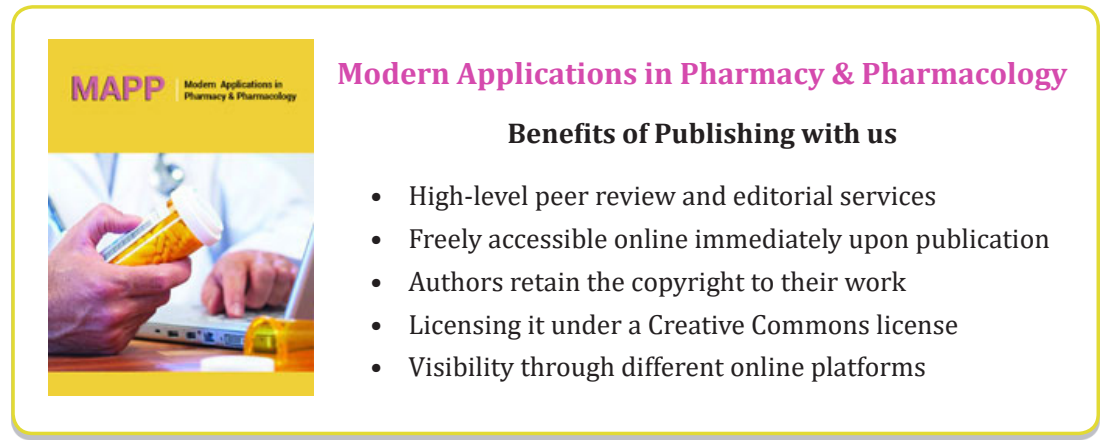

